\title{
Cooperation in Random Access Networks: Protocol Design and Performance Analysis
}

\author{
Amr A. El-Sherif and K. J. Ray Liu
}

\begin{abstract}
This paper tries to answer the questions of how to enable cooperative communications in random access networks. And, since cooperation introduces extra transmissions in the channel, what are the benefits and possible tradeoffs associated with cooperation? To answer these questions, a novel cooperative protocol for wireless networks based on IEEE 802.11 random access protocol is proposed. Cooperation is achieved through the deployment of a relay node that intelligently access the wireless medium without imposing any penalties in terms of increased collision probability. To help other network nodes, relay stores packets in its queue, and accesses the channel after each transmission attempt made by any network node. Using this scheme, relay is guaranteed uncontested access to the channel. To capture the interactions between different network nodes and the relay, the network is modeled using Markov chains in conjunction with queuing analysis. Throughput and delay performances of the proposed protocol are characterized and compared to CSMA/CA without cooperation. The results demonstrate that significant gains are achieved by the proposed cooperative protocols.
\end{abstract}

Index Terms-Cooperative communications, random access, queuing theory.

\section{INTRODUCTION}

$\mathbf{C}$ OOPERATIVE communications is a concept that has been recently introduced to improve reliability and throughput in wireless networks [1], [2], [3]. The broadcast nature of the wireless medium is the key property that allows for cooperation among networking nodes. In cooperative networks, users cooperate by relaying each others' message over multiple paths. At the destination, the original and relayed signals are combined to generate a signal with better quality, creating a new form of diversity which can significantly improve the system performance and robustness.

Some works have focused on the impact and implementation of cooperation at higher network layers. A cognitive multiple access protocol was proposed in [4]. The protocol benefits from source burstiness to enable cooperation by allowing a relay to utilize the nodes' periods of silence. In [5], the authors have proposed a cooperative multiple access protocol for speech networks. The protocol enables cooperation by exploiting the relatively long silent periods typical in speech communications. Cooperation in random access networks has been considered in [6], where a distributed version of network diversity multiple-access (NDMA) [7] protocol was proposed. The main focus of [6] and [7] is on collision resolution using conventional source separation techniques at the destination, the corrupted packets are retrieved.

Manuscript received 20 January 2011; revised 20 June 2011.

A. A. El-Sherif is with the Department of Electrical Engineering, Alexandria University, Alexandria 21544, Egypt (e-mail: amr.elsherif@ieee.org).

K. J. Liu is with the Department of Electrical and Computer Engineering, University of Maryland, College Park, MD 20742, USA (e-mail: kjrliu@umd.edu).

Digital Object Identifier 10.1109/JSAC.2012.121013.
To enable cooperation in a wireless network, transmissions among the cooperating nodes are generally needed in order to coordinate their actions. In large random access networks without centralized scheduler like in IEEE 802.11 networks [8], these extra transmissions will increase the number of packet collisions. As such it is not clear if there is any benefit of using physical layer cooperation in this case.

To answer the question of how to enable cooperation in a random access network without the increased number of packet collisions, we begin by proposing a cooperative protocol in which a relay node is deployed to help different network nodes forward their packets to the access point (AP). In order not to increase the collision probability, the relay intelligently accesses the wireless medium at times when it is guaranteed that no other node is accessing the medium. In the IEEE 802.11 protocol and the CSMA/CA protocol, on which it is based, after each packet transmission, and irrespective of the outcome of this transmission, all nodes wait for a random amount of time before making a transmission attempt. By allowing the relay to access the channel immediately after each transmission attempt, it is guaranteed to have an uncontested access to the wireless medium.

In a network operating as described above, all nodes's queues are interacting, i.e., the service process of a given queue depends on the state of all other queues (whether they are empty or not). Interaction between queues is mainly because of the possible collisions that occur if multiple nodes try to access the channel at the same instant. And, for the relay, its own ability to access the channel is dependent on other nodes' transmissions.

In order to capture this queue interaction, and to be able to analyze the performance of our protocol, two coupled Markov models are used to describe the operation of the relay and other network nodes. Both Markov models together are able to completely describe the dynamics of the network and interactions between different nodes. Moreover, queuing analysis is used to analyze the delay performance of the network, and the model is validated through simulation. The results presented reveal significant gains in terms of network throughput, delay, and the number of supported nodes, due to cooperation and our proposed protocol. Furthermore, it is shown that, by virtue of the protocol design, collision probability has decreased rather than the expected increase due to extra transmissions on the channel.

The rest of the paper is organized as follows. In section II, the channel and network models are presented. The proposed cooperation protocol is presented in section III. Markov and queuing models are developed and analyzed in sections IV and $\mathrm{V}$, respectively. Network throughput and delay are characterized in section VI. Section VII presents the numerical 
results and their discussions. Finally, the paper is concluded in section VIII.

\section{Channel And System Models}

\section{A. Channel model}

A Rayleigh fading channel model is considered, the signal received at the access point or the relay is modeled as

$$
y_{i j}=\sqrt{G r_{i j}^{-\gamma}} h_{i j} x+\eta_{j}
$$

where $i$ is the source index, $j \in\{A, R\}$ is the access point or the relay index, $x$ is the transmitted signal, $G$ is the transmission power, assumed to be the same for all nodes, $r_{i j}$ denotes the distance between source node $i$ and its destination $j, \gamma$ is the path loss exponent, $h_{i j}$ the channel fading coefficients, modeled as zero-mean complex Gaussian random variables with unit variance, and $\eta_{j}$ is an additive noise term at the destination, modeled as zero-mean complex Gaussian random variable with variance $N_{0}$. We assume that the channel coefficients are constant for the duration of the transmission of one packet. In this work, without loss of generality, we only considered the case of a symmetric network, where all the inter-user channels are assumed to be statistically identical. This is possible for example when nodes are grouped in a small cluster at fixed distance from the access point.

Success and failure of packet reception is characterized by outage events and outage probabilities. The outage probability is defined as the probability that the Signal to Noise Ratio (SNR) at the receiver is less than a given SNR threshold $\beta$. For the channel model in (1) the probability of outage can be written as,

$$
P_{i j}^{\text {out }}=\operatorname{Pr}\left\{\left|h_{i j}\right|^{2}<\frac{\beta N_{0} r_{i j}^{\gamma}}{G}\right\}=1-\exp \left(-\frac{\beta N_{0} r_{i j}^{\gamma}}{G}\right) .
$$

\section{B. Network Model}

Our work in this paper focuses on wireless networks in which all nodes and the AP are within communication range from each other. In other words, we are only considering a single hop wireless network where each node is communicating only with the AP. Based on this assumption, this single hop network does not suffer from the hidden node problem since any node can overhear all other nodes. Without loss of generality, this paper also focuses on the single relay case. Due to space limitation the case of a multi-hop network with multiple relays is not considered in this paper. Extension of our cooperation protocol design to the multiple-hop network case, how to deal with hidden nodes and the effect of cooperation on routing will be the subject of a future work.

\section{IEEE 802.11 DCF Operation}

The distributed coordination function (DCF) is the fundamental medium access mechanism in the IEEE 802.11 protocol [8]. It is a random access scheme based on the CSMA/CA (Carrier Sense Multiple Access with Collision Avoidance) with binary slotted exponential backoff. As depicted in fig. 1 a node with a packet to transmit invokes the carrier sensing mechanism to determine the busy/idle state of the channel. If

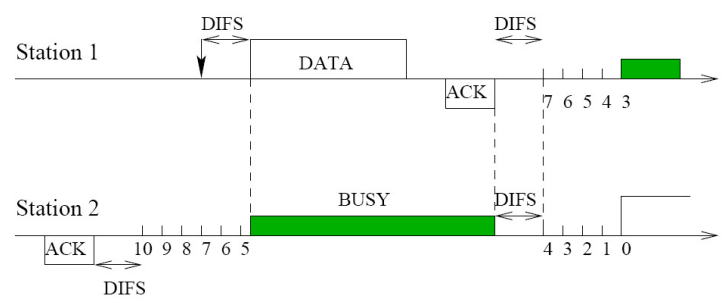

Fig. 1. DCF basic access mechanism; numbers in figure represent node's backoff timer.

the channel is sensed to be idle for a period of time equal to a Distributed Inter-Frame Space (DIFS), the node proceeds with packet transmission. Otherwise, it persists to monitor the channel until it is measured idle for a DIFS. The node then defers for a randomly selected backoff interval, initializing its random backoff timer, which is decremented as long as the channel is sensed idle and is frozen when a transmission is detected.

The time immediately following an idle DIFS is slotted, and a node is allowed to transmit at the beginning of a slot time if its backoff timer reaches zero. The slot duration, $\sigma$, is set equal to the time needed for any node to detect the transmission from any other node. It depends on the physical layer, and it accounts for the propagation delay, and the time needed to detect a busy channel.

The random backoff interval is uniformly chosen in the range $(0, w-1)$. The value $w$ is called the contention window. At the first transmission attempt, $w$ is set equal to a minimum contention window value $C W_{\min }$. After each unsuccessful transmission $w$ is doubled, up to a maximum value $C W_{\max }=2^{m} C W_{\min }, m$ is the maximum backoff stage. Once $w$ reaches $C W_{\max }$, it remains at this value until it is reset to $C W_{\min }$ after a successful transmission.

To signal the successful packet reception, an ACK is transmitted by the destination. The ACK is transmitted after a period of time called short inter-frame space (SIFS). As the SIFS is shorter than a DIFS, no other station is able to detect the channel idle for DIFS until the end of the ACK. If the transmitting node does not receive the ACK within a specified ACK_Timeout, the packet is assumed to be lost and the node reschedules the packet transmission according to the given backoff rules.

\section{RANDOM ACCESS COOPERATION PROTOCOL}

Inherent wireless channel fading and transmission errors have a significant impact on the network's performance [9]. In wireless networks, nodes are unable to detect collisions by hearing their own transmission. Therefore, there is no differentiation between a packet loss due to a collision, and a packet loss due fading. Therefore, a source node will deal with a wireless channel induced packet loss in the same way it deals with a collision induced one. Hence, doubling its contention window and waiting for a random amount of time before reattempting transmission. As a result of invoking the backoff procedure in a non-congested channel, the network will suffer from an increased delay and lower achievable throughput [10].

To combat the wireless channel impairments leading to these problems, we propose the deployment of a cooperative 
relay node into the coverage area of the wireless network. The cooperative relay node will help combat the channel fading through the introduction of spatial diversity into the network. Relay node will help source nodes forward their packets by operating in an incremental decode-and-forward mode [1]. In this mode, in case of a packet loss at the access point, the relay attempts to decode the received packet, and in case of successful detection at the relay, it forwards a regenerated version of the packet to the AP.

The relay makes use of the AP's ACK packet to know if a packet is successfully received by the AP. In case the relay successfully receives a packet, but the AP does not receive that packet (ACK_Timeout occurs), the relay stores it in its queue and sends an ACK packet over the channel to inform other nodes that the packet was received successfully. Upon receiving the relay's ACK packet, the packet owner drops it from its queue and the delivery of that packet becomes the relay's responsibility. Because of the relay's ACK packet, the node with the lost packet will reset its contention window $C W_{\min }$.

The challenging part in the design of our cooperation protocol is to enable the relay to gain access to the wireless channel without increasing the number of collisions, and hence rendering its existence useless. To deal with this issue we propose the following relay channel access protocol:

- Following a transmission attempt from one or more source nodes (outcome of the transmission attempt is irrelevant), the relay node attempts to transmit the packet at the head of its queue immediately after the AP's ACK, or after the ACK_Timeout.

- For the relay not to be totally dependent on other nodes' transmission attempts, the relay also maintains a single stage backoff counter with contention window size $C W_{r}$

- When the relay's backoff counter reaches zero, it will attempt to transmit the packet at the head of its queue like other network nodes.

- Like other network nodes, the relay will invoke the backoff procedure after each transmission attempt, the only difference is that the relay has a single backoff stage as opposed to $m$ stages for other nodes.

By accessing the channel after each transmission attempt the relay has the ability to serve the packets in its queue without causing any collision.

\section{MARKov MOdels AND ANALYSIS}

A number of models have been proposed to study the performance of IEEE 802.11 DCF in the saturated [11], [12], unsaturated [13], [14] traffic conditions, and in the presence of channel impairments [10]. To analyze the performance of the proposed cooperative protocol, we start from the discretetime Markov model for non-saturated sources developed in [14], and incorporate the channel effects and relay operation into the model. We consider two separate Markov chains, the first chain models source nodes while the second models the relay node.

We assume that the network consists of $N$ contending nodes in addition to the relay node. Each node has an infinite queue to store packets. Each node receives packets from upper layer based on a Poisson arrival process with arrival

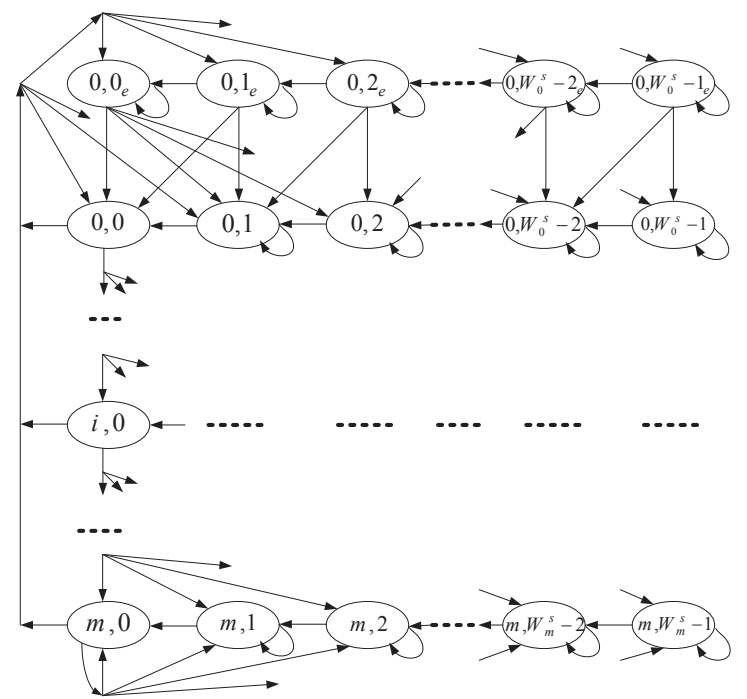

Fig. 2. Source node's Markov model.

rate $\lambda_{s}$ packets/sec (The super(sub)script $\mathrm{s}$ or $\mathrm{r}$ are used to differentiate between source node and relay parameters), and fixed packet size $L$. The queuing model used will be discussed in details in section $\mathrm{V}$.

\section{A. Source Nodes}

Fig. 2 represents the discrete-time Markov chain used to model the operation of source nodes. Each node is modeled by a pair of integers $(i, k)$. The backoff stage $i$, starts at 0 at the first attempt to transmit a packet and is increased by 1 every time a transmission attempt fails, up to a maximum value $m$. It is reset after a successful transmission. At any backoff stage $i \in[0, m]$, the backoff counter, $k$, is initially chosen uniformly between $\left[0, W_{i}^{s}-1\right]$, where $W_{i}^{s}=2^{i} W_{0}^{s}, \quad 0 \leq i \leq m$, is the range of the counter, and $W_{0}^{s}$ is the parameter $\mathrm{CW}_{\min }$ specified in the IEEE 802.11 standard. The backoff counter is decremented by 1 in each idle time slot of duration $\sigma$, and the node transmits when the backoff counter $k=0$.

States $(0, k)_{e}, k \in\left[0, W_{0}^{s}-1\right]$ are introduced to represent the state of the node when it has an empty queue after a successful transmission. Note that $i=0$ in these states because if $i>0$ then a failed transmission should have occurred, so a packet must be awaiting.

The fundamental assumption in our model is that, at each transmission attempt, and regardless of the number of retransmissions suffered, each packet fails with a constant and independent probability, $P_{f}^{s}$ or $P_{f}^{r}$, for the source nodes or relay node, respectively [11], [14].

Let $\tau_{s}$ and $\tau_{r}$ be the probability that a source node or the relay transmit in a given slot, respectively. Now we are ready to write the Markov chain's transition probabilities, for $0 \leq$ $i \leq m$

$$
\begin{array}{ll}
P\{(i, k) \mid(i, k+1)\}=P_{i}, & 0 \leq k \leq W_{i}^{s}-2 \\
P\{(i, k) \mid(i, k)\}=\left(1-P_{i}\right), & 0 \leq k \leq W_{i}^{s}-1 \\
P\{(0, k) \mid(i, 0)\}=\frac{\left(1-q_{s}\right)\left(1-P_{f}^{s}\right)}{W_{0}^{s}}, & 0 \leq k \leq W_{0}^{s}-1 \\
P\left\{(0, k)_{e} \mid(i, 0)\right\}=\frac{q_{s}\left(1-P_{f}^{s}\right)}{W_{0}^{s}}, & 0 \leq k \leq W_{0}^{s}-1
\end{array}
$$


where $q_{s}$ is the probability that the node's queue is empty upon a departure (see section V). $P_{i}$ is the probability that the channel is sensed idle by the source node (i.e., all the remaining $N-1$ source nodes and the relay node are not attempting to transmit), and is given by $P_{i}=\left(1-\tau_{s}\right)^{N-1}\left(1-\tau_{r}\right)$. A source node's transmission attempt is considered successful if the channel is idle (i.e., no collision) and either the AP or the relay correctly receive the transmitted packet, in other words if either the source-AP or the source-relay channel is not in outage. Let $P_{f}^{s}$ be the probability of a failed transmission attempt, since a failed transmission is the complement event of a successful transmission (a transmission is successful if the channel is idle and either the source-AP link or the sourcerelay link is not in outage), then $P_{f}^{s}=1-P_{i}\left(1-P_{s A}^{\text {out }} P_{s R}^{\text {out }}\right)$, where $P_{s A}^{\text {out }}$ and $P_{s R}^{\text {out }}$ are the outage probabilities of the source-AP and source-relay links, respectively.

The first and second equations in (3) accounts for the fact that at the beginning of each idle slot time the backoff counter is decremented by one, and that the counter remains at its current state if the channel is not idle. The third and forth equations account for the fact that following a successful packet transmission, backoff stage $i$ is reset to 0 , and thus the backoff is initially uniformly chosen in the range $\left[0, W_{0}^{s}-1\right]$.

In case of an unsuccessful transmission at backoff stage $i-1$, the backoff stage is increased, and the new initial backoff counter is initially chosen in the range $\left[0, W_{i}^{s}-1\right]$. Once the backoff stage reaches the value $m^{s}$, it is not increased in subsequent packet transmissions, then we have

$$
\begin{aligned}
& P\{(i, k) \mid(i-1,0)\}=P_{f}^{s} / W_{i}^{s}, \\
& P\{(m, k) \mid(m, 0)\}=P_{f}^{s} / W_{m}^{s} .
\end{aligned}
$$

Given that the node's queue is empty and the chain is in state $(0, k)_{e}$, in case of a packet arrival, the backoff counter is decremented and the chain makes a transition into the $(0, k-1)$ state if the channel is idle, and to state $(0, k)$ if the channel is not idle. In the case of an idle channel but no packets arrives to the queue the chain transits into $\left(0,(k-1)_{e}\right)$. When the backoff timer reaches zero, the node remains in state $\left(0,0_{e}\right)$ as long as the queue is empty. If a packet arrives, then the node moves into state $(0, k)$, where $k$ is uniformly chosen in the range $\left[0, W_{0}^{s}-1\right]$. Therefore we have

$$
\begin{aligned}
& P\left\{(0, k)_{e} \mid 0,(k+1)_{e}\right\}=P_{i}\left(1-a_{i}\right), \quad 0 \leq k \leq W_{0}^{s}-2 \\
& P\left\{(0, k) \mid\left(0,(k+1)_{e}\right)\right\}=P_{i} a_{i}, \quad 0 \leq k \leq W_{0}^{s}-2 \\
& \left.P\left\{\left(0, k_{e}\right) \mid\left(0, k_{e}\right)\right)\right\}=\left(1-P_{i}\right)\left(1-a_{b}\right), \quad 0 \leq k \leq W_{0}^{s}-1 \\
& P\left\{(0, k) \mid\left(0, k_{e}\right)\right\}=\left(1-P_{i}\right) a_{b}, \quad 0 \leq k \leq W_{0}^{s}-1 \\
& P\left\{\left(0,0_{e}\right) \mid\left(0,0_{e}\right)\right\}=1-\left(P_{i} a_{i}+\left(1-P_{i}\right) a_{b}\right), \\
& 0 \leq k \leq W_{0}^{s}-1 \\
& P\left\{(0, k) \mid\left(0,0_{e}\right)\right\}=\frac{P_{i} a_{i}+\left(1-P_{i}\right) a_{b}}{W_{0}^{s}}, \quad 0 \leq k \leq W_{0}^{s}-1
\end{aligned}
$$

where $a_{i}$ and $a_{b}$ are the probabilities of at least one packet arrival during an idle or a busy slot, respectively. From the Poisson arrival assumption, these probabilities are given by $a_{i}=1-e^{-\lambda_{s} \sigma}$ and $a_{b}=1-e^{-\lambda_{s} T_{b}}$, where $\sigma$ is the idle slot duration, and $T_{b}$ the busy slot duration (for simplicity we neglect the difference in durations between successful and

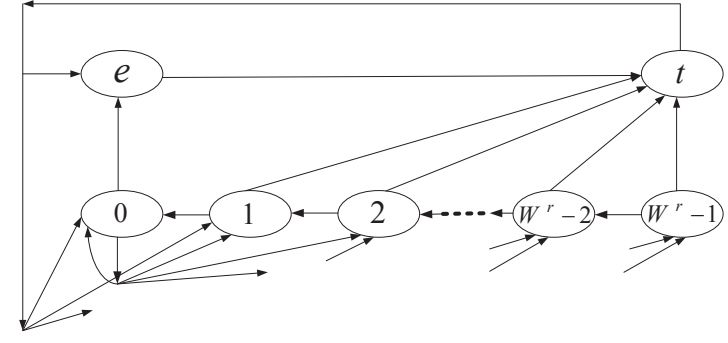

Fig. 3. Relay node's Markov model.

unsuccessful transmission attempts). Typically, $\sigma=20 \mu \mathrm{s}$, and $T_{b}=2160.4 \mu \mathrm{s}$, based on $11 \mathrm{Mbps}$ channel rate and packet size $L=2312$ octets [8].

Let $\pi_{s}(i, k)$ denote the stationary probability of being in state $(i, k)$. To solve for the stationary distribution of this Markov chain we used balance equations [15] to find expressions for all the stationary probabilities as a function of $\pi_{s}(0,0)$. Imposing the normalization condition $\sum_{i=0}^{m} \sum_{k=0}^{W_{i}^{s}-1} \pi_{s}(i, k)+\sum_{k=0}^{W_{0}^{s}-1} \pi_{s}(0, k)_{e}=1$, we can calculate $\pi_{s}(0,0)$, hence, all the steady state probabilities. Full derivation of the closed form expressions is omitted because of the limited space, however a similar procedure to our analysis can be found in [11].

Finally, since a node will make a transmission attempt in a given slot time if the Markov chain is in state $\pi_{s}(i, 0)$ for $i \in[0, m]$, then, $\tau_{s}$, the probability that a source node makes a transmission attempt in a given slot time can be expressed as $\tau_{s}=\sum_{i=0}^{m} \pi_{s}(i, 0)$.

\section{B. Relay Node}

A relay node operating as described in section III will be modeled using the Markov chain model of Fig. 3. The model has a single backoff stage represented by states $k \in\left[0, W^{r}-\right.$ $1]$. The backoff counter is uniformly chosen in that range, and the relay makes a transmission attempt when in state 0 . The relay node makes a transition to state $e$ if its queue becomes empty after a successful transmission. Finally, the chain is in state $t$ when the relay is attempting to transmit following a busy channel.

Again we have the assumption that, at each transmission attempt, and regardless of the number of re-transmissions suffered, each packet fails with a constant and independent probability $P_{f}^{s}$ or $P_{f}^{r}$, for the source nodes or relay node, respectively [14], [11]. Now we are ready to write the Markov chain's transition probabilities. At the beginning of each idle slot time, the backoff counter is decremented, then

$$
P\{k \mid k+1\}=P_{i}, \quad 0 \leq k \leq W^{r}-1,
$$

where $P_{i}$ is the probability that the channel is sensed idle by the relay node (i.e., all $N$ source nodes are not attempting to transmit), and is given by $P_{i}=\left(1-\tau_{s}\right)^{N}$.

Since the relay Markov chain has a single backoff stage, following an unsuccessful transmission attempt or a successful attempt that leaves the relay queue non-empty, the backoff counter is initially uniformly chosen in the range $\left[0, W^{r}-1\right]$. 
Therefore

$$
\begin{array}{ll}
P\{k \mid 0\}=\frac{\left(1-q_{r}\right)\left(1-P_{f}^{r}\right)}{W^{r}}+\frac{P_{f}^{r}}{W^{r}}, & 0 \leq k \leq W^{r}-1 \\
P\{k \mid t\}=\frac{\left(1-q_{r}\right)\left(1-P_{f}^{\prime r}\right)}{W^{r}}+\frac{P_{f}^{\prime r}}{W^{r}}, & 0 \leq k \leq W^{r}-1
\end{array}
$$

where $q_{r}$ is the probability that a departing packet will leave the relay queue empty, $P_{f}^{r}$ is the probability of a failed relay transmission attempt out of state 0 (failure due to collision or channel error), and $P_{f}^{\prime r}$ is the probability of a failed transmission attempt out of state $t$ (failure can be caused only by channel errors), and are given by $P_{f}^{r}=1-\left(1-\tau_{s}\right)^{N}\left(1-P_{R A}^{o u t}\right)$ and $P_{f}^{\prime r}=P_{R A}^{o u t}$, where $P_{R A}^{o u t}$ is the outage probability of the relay-AP link.

A successful transmission that leaves the relay queue empty leads to a transition to state $e$, then we have

$$
\begin{aligned}
& P\{e \mid 0\}=q_{r}\left(1-P_{f}^{r}\right) \\
& P\{e \mid t\}=q_{r}\left(1-P_{f}^{\prime r}\right) .
\end{aligned}
$$

Transitions into state $t$ occur when the relay attempts to transmit a packet immediately after any transmission attempt on the channel, thus

$$
\begin{aligned}
& P\{t \mid e\}=N \tau_{s}\left(1-\tau_{s}\right)^{N-1} P_{s A}^{\text {out }}\left(1-P_{s R}^{\text {out }}\right)=a \\
& P\{t \mid k\}=1-P_{i}, \quad 0<k \leq W^{r} .
\end{aligned}
$$

The first equation of (9) accounts for the case when the relay queue is empty and that a source node transmission lead to a packet arrival at the relay, then the relay will immediately forward that packet. The second equation of (9) is for the case when the relay queue is not empty, thus, the relay will transmit after a busy period irrespective of the outcome of that period.

Let $\pi_{r}(k)$ denote the stationary probability of being in state $k$. Similar to the source nodes, Markov chain, the stationary distribution of the relay's Markov chain can be obtained by expressing all the stationary probabilities as a function of $\pi_{r}(0)$ and imposing the normalization condition $\sum_{k=0}^{W^{r}-1} \pi_{r}(k)+\pi_{r}(e)+\pi_{r}(t)=1$, to calculate $\pi_{r}(0)$, hence, all the steady state probabilities.

Finally, the probability $\tau_{r}$, that the relay node makes a transmission attempt in a given slot, is given by $\tau_{r}=\pi_{r}(0)$. Note that transmissions out of state $t$ are not included in this probability calculation since these transmission events are not initiated by backoff counter timeout, and hence cannot result in collisions with source node transmissions.

\section{QUEUING ANALYSiS}

We assumed that each node receives packets from upper layer based on a Poisson arrival process with arrival rate $\lambda_{s}$ packets/sec, and fixed packet size $L$. Packet processing at each node can be seen as a single server with service rate $\mu_{s}$, which depends on the channel access mechanism, the interaction between different nodes, and the channel statistics. Therefore, node queues can be modeled as $\mathrm{M} / \mathrm{G} / 1$ queues.

The relay node can also be seen as a single server system. However, in the case of the relay, both the arrival rate, $\lambda_{r}$, and the service rate, $\mu_{r}$, are dependent on the channel access mechanism, interaction between different nodes and the relay, channel statistics, and the number of source nodes and their arrival rates. For mathematical tractability, we will model the relay as an M/G/1 queuing system. Simulation results will later show that this is in fact a good approximation to the behavior of relay queue.

Since for any queueing system with single (in contrast to batch) arrivals and departures, it holds that the queue length seen by an arriving customer is equal to its length left by a departure customer. Furthermore, using the PASTA (Poisson Arrivals See Time Averages) property [15] associated with Poisson arrivals, the queue length at an arbitrary time equals the queue length seen by an arriving customer. This enables us to calculate the probability that a node queue is empty as follows [16], $q_{s}=1-\frac{\lambda_{s}}{\mu_{s}}$, similarly, for the relay queue $q_{r}=1-\frac{\lambda_{r}}{\mu_{r}}$.

\section{A. Source Nodes Service Rate}

Service time, $S_{s}$, of a packet is defined as the interval between the time the packet becomes at the head of the transmission queue and the time the packet is acknowledged for correct reception (reception by either the AP or the relay node). We will use the probability generating function (PGF) [15] to characterize the discrete probability distribution of the service time, $S_{s}$.

Time spent in the backoff counter decrements constitutes the first component of a packet's service time. For the backoff counter to decrement by 1 (i.e., the Markov chain of Fig. 2 makes a transition from state $(i, k)$ to state $(i, k-1))$, a node will, in general, spend $j$ busy slots and a single idle slot (at which transition occurs) at each step of the counter. We should also note that, because of the way the relay accesses the channel, there will be two types of busy periods: (i) a period of duration $T_{b}$, if the relay does not access the channel immediately after a source node transmission. This occurs if the relay queue is empty, and the source node transmission does not result in a packet arrival at the relay (i.e., transmission resulted in a collision, a successful reception at the AP, or a failure to reach both the AP and the relay). From the point of view of the source node of interest, this event has the probability

$$
\begin{aligned}
& P_{b 1}=\pi_{r}(e) \\
& {\left[1-\left(1-\tau_{s}\right)^{N-1}-(N-1) \tau_{s}\left(1-\tau_{s}\right)^{N-2} P_{s A}^{o u t}\left(1-P_{s R}^{o u t}\right)\right] .}
\end{aligned}
$$

(ii) A busy period of duration $2 T_{b}$, if the relay accesses the channel immediately after a source node transmission (either the relay queue is not empty, or the source node transmission resulted in an arrival at the relay). A given source node will observe this event with probability $P_{b 2}=\pi_{r}(e)(N-1) \tau_{s}(1-$ $\left.\tau_{s}\right)^{N-2} P_{s A}^{\text {out }}\left(1-P_{s R}^{\text {out }}\right)+\left[1-\left(1-\tau_{s}\right)^{N-1}\right] \sum_{k=1}^{W^{r}-1} \pi_{r}(k)$.

The PGF characterizing the distribution of the time spend at each counter step could be written as

$$
\begin{aligned}
F(z) & =P_{i} z^{\sigma} \sum_{i=0}^{\infty} \sum_{j=0}^{i}\left(\begin{array}{l}
i \\
j
\end{array}\right)\left(P_{b 1} z^{T_{b}}\right)^{j}\left(P_{b 2} z^{2 T_{b}}\right)^{i-j} \\
& =\frac{P_{i} z^{\sigma}}{1-P_{b 1} z^{T_{b}}-P_{b 2} z^{2 T_{b}}} .
\end{aligned}
$$


The next component is the time spent in a backoff stage $i$ before making a transmission attempt (i.e., before the backoff counter reaches zero). At stage $i$ the counter is initialized uniformly in the range $k \in\left[0, W_{i}^{s}-1\right]$, therefore the distribution of the time spent in stage $i$ is characterized by the PGF, $F_{i}(z)=\sum_{k=0}^{W_{i}^{s}-1} \frac{F^{k}(z)}{W_{i}^{s}}$.

Finally, the PGF for the service time $S_{s}$ can be written as

$$
\begin{array}{r}
G_{s}(z)=\left(1-P_{f}^{s}\right) z^{T_{b}}\left[\sum_{i=0}^{m-1}\left(P_{f}^{s} z^{T_{b}}\right)^{i} \prod_{j=0}^{i} F_{j}(z)\right. \\
\left.+\left(P_{f}^{s} z^{T_{b}}\right)^{m} \prod_{j=0}^{m} F_{j}(z) \sum_{i=0}^{\infty}\left(P_{f}^{s} z^{T_{b}}\right)^{i} F_{m}^{i}(z)\right] \\
=\left(1-P_{f}^{s}\right) z^{T_{b}}\left[\sum_{i=0}^{m-1}\left(P_{f}^{s} z^{T_{b}}\right)^{i} \prod_{j=0}^{i} F_{j}(z)\right. \\
\left.+\frac{\left(P_{f}^{s} z^{T_{b}}\right)^{m} \prod_{j=0}^{m} F_{j}(z)}{1-P_{f}^{s} z^{T_{b}} F_{m}^{i}(z)}\right],
\end{array}
$$

where the term outside the brackets accounts for the busy slot in which the packet is successfully delivered, the first term inside the brackets accounts for the possible number of failures a packet encounters (hence, the number of backoff stages it goes through), its composed of the time spent in the backoff counter decrements and the time spent transmitting the packet. Finally, the second term accounts for the amount of time spent at the maximum backoff stage $m$, which is decomposed into the time spent to reach this state, the time for counter decrements, and the time for packet transmission. The service rate can then be calculated by differentiating $G_{s}(z)$ and setting $z=1, \mu_{s}^{-1}=E\left[S_{s}\right]=\left.\frac{d G_{s}(z)}{d z}\right|_{z=1}$.

\section{B. Relay Node Arrival Rate}

The time, $A_{r}$, between packet arrivals to the relay queue is composed of the following components: (i) Idle periods in which no node (source or relay) is transmitting. This periods have a length $\sigma$ and probability $P_{i}=\left(1-\tau_{s}\right)^{N}\left(1-\tau_{r}\right)$. (ii) Busy periods of duration $T_{b}$, which occur if the relay queue is empty and the transmission attempt does not result in an arrival at the relay. This occurs with probability

$$
\begin{aligned}
& P_{b 1}=\pi_{r}(e) \\
& {\left[1-\left(1-\tau_{s}\right)^{N-1}-(N-1) \tau_{s}\left(1-\tau_{s}\right)^{N-2} P_{s A}^{o u t}\left(1-P_{s R}^{o u t}\right)\right] .}
\end{aligned}
$$

(iii) Busy periods of duration $2 T_{b}$ not resulting in a relay arrival, which occur if the relay queue is not empty when a source node makes a transmission attempt. Thus has a probability

$$
\begin{aligned}
& P_{b 2}= \\
& {\left[1-\left(1-\tau_{s}\right)^{N-1}-(N-1) \tau_{s}\left(1-\tau_{s}\right)^{N-2} P_{s A}^{\text {out }}\left(1-P_{s R}^{\text {out }}\right)\right]} \\
& \sum_{k=1}^{W^{r}-1} \pi_{r}(k) .
\end{aligned}
$$

(iv) Finally, a busy period during which a packet enters the relay queue. This will always have a duration $T_{b}$, and has a probability $P_{a}=\left[\pi_{r}(e)+\sum_{k=1}^{W^{r}-1} \pi_{r}(k)\right](N-1) \tau_{s}(1-$ $\left.\tau_{s}\right)^{N-2} P_{s A}^{\text {out }}\left(1-P_{s R}^{\text {out }}\right)$.

Given the above mentioned probabilities, we can write the PGF of $A_{r}$ as follows,

$$
\begin{aligned}
G_{a}(z)= & P_{a} z^{T_{b}} \sum_{i=1}^{\infty} \sum_{j=1}^{i} \sum_{k=0}^{i-j}\left[\frac{i !}{j ! k !(i-j-k) !}\right. \\
& \left.\cdot\left(P_{i} z^{\sigma}\right)^{j}\left(P_{b 1} z^{T_{b}}\right)^{k}\left(P_{b 2} z^{2 T_{b}}\right)^{i-j-k}\right] .
\end{aligned}
$$

The arrival rate can then be calculated by differentiating $G_{a}(z)$ and setting $z=1, \lambda_{r}^{-1}=E\left[A_{r}\right]=\left.\frac{d G_{a}(z)}{d z}\right|_{z=1}$.

\section{Relay Node Service Rate}

Similar to the way the source nodes service rate was calculated, we will start the calculation of the relay node service rate by defining the different components that constitute a packet's service time $S_{r}$. We note that, as opposed to source nodes, the relay can leave the backoff stage after any source node's transmission attempt on the channel (Fig. 3). Therefore, the time the packet at the head of the queue spends in the backoff stage can be split into two components: (i) The time before the backoff counter (initialized uniformly between 0 and $W^{r}-1$ ) reaches 0 , which in the relay case is composed only of idle slots. The PGF characterizing the distribution of that time is then given by

$$
F_{0}=\sum_{k=0}^{W^{r}-1} \frac{P_{i}^{k} z^{k \sigma}}{W^{r}} .
$$

(ii) The time spent in the backoff stage before the Markov chain reaches state $t$, which is composed of a single busy period and a maximum of $W^{r}-1$ idle slots. The PGF characterizing the distribution of that time is then given by

$$
F_{t}=\left(1-P_{i}\right) z^{T_{b}} \sum_{k=0}^{W^{r}-2} \frac{P_{i}^{k} z^{k \sigma}}{W^{r}} .
$$

Finally, let the probability that a packet enters relay queue be $a=N \tau_{n}\left(1-\tau_{n}\right)^{N-1} P_{s A}^{\text {out }}\left(1-P_{s R}^{\text {out }}\right)$, the PGF for the service time $S_{r}$ can be written as

$$
\begin{array}{r}
G_{r}(z)=\pi_{r}(e) a\left(1-P_{f}^{\prime r}\right) z^{T_{b}}+\left(a P_{f}^{\prime r} z^{T_{b}}+\sum_{k=0}^{W^{r}-1} \pi_{r}(k)\right) \\
\cdot\left[\left(F_{0}(z)\left(1-P_{f}^{r}\right) z^{T_{b}}+F_{t}(z)\left(1-P_{f}^{\prime r}\right) z^{T_{b}}\right)\right. \\
\left.\cdot \sum_{i=0}^{\infty} \sum_{j=0}^{i}\left(\begin{array}{l}
i \\
j
\end{array}\right)\left(F_{0}(z) P_{f}^{r} z^{T_{b}}\right)^{j}\left(F_{t}(z) P_{f}^{\prime r} z^{T_{b}}\right)^{i-j}\right],
\end{array}
$$

which accounts for the case when a packet is immediately

served by the relay after it enters the queue (if queue was empty at packet arrival), the possible number of failures a packet encounters getting transmitted from either state 0 or state $t$, and finally, the periods at which the packet is 
delivered successfully. The service rate can then be calculated by differentiating $G_{r}(z)$ and setting $z=1, \mu_{r}^{-1}=E\left[S_{r}\right]=$ $\left.\frac{d G_{r}(z)}{d z}\right|_{z=1}$.

\section{Vi. Performance Measures}

\section{A. Network Throughput}

Let $S$ be the normalized network throughput, defined as the fraction of time the channel is used to successfully transmit payload bits to the AP, which can be expressed as $S=\frac{P_{s} \cdot T_{p}}{T_{s}}$, where $P_{s}$ is the probability of a successful transmission to the AP (by source or relay nodes), $T_{p}$ is the time to transmit the payload part of a packet, of course this is less than $T_{b}$, the total transmission time of a packet including the headers and the AP ACK packet. And $T_{s}$ is the expected slot duration.

To calculate the probability $P_{s}$, we identify the events that result in a successful packet delivery to the AP, which are: (i) If the relay queue is empty, a source node successfully transmits a packet to the AP, or that packet fails to reach the AP but was successfully received and forwarded by the relay. This event has a probability $P_{s}^{1}=\pi_{r}(e)\left[N \tau_{s}(1-\right.$ $\left.\left.\tau_{s}\right)^{N-1}\left(\left(1-P_{s A}^{\text {out }}\right)+P_{s A}^{\text {out }}\left(1-P_{s R}^{\text {out }}\right)\left(1-P_{R A}^{\text {out }}\right)\right)\right]$. (ii) If the relay queue is not empty, a source node transmission fails to reach the AP (due to fading or collision), and the relay successfully transmits the packet at the head of its queue to the AP. This occurs with probability $P_{s}^{2}=$ $\left[1-\left(1-\tau_{s}\right)^{N}-N \tau_{s}\left(1-\tau_{s}\right)^{N-1}\left(1-P_{s A}^{\text {out }}\right)\right] \sum_{k=0}^{W^{r}} \pi_{r}(k)$. (iii) If the relay queue is not empty and both source node transmission and the immediately following relay node transmission were successful. This occurs with probability $P_{s}^{3}=N \tau_{s}\left(1-\tau_{s}\right)^{N-1}\left(1-P_{s A}^{o u t}\right)\left(1-P_{R A}^{\text {out }}\right) \sum_{k=0}^{W^{r}} \pi_{r}(k)$. (iv) The relay succeeds in transmitting a packet when its backoff counter reaches 0 , which has a probability $P_{s}^{4}=\tau_{r}\left(1-\tau_{s}\right)^{N}\left(1-P_{R A}^{\text {out }}\right)$. Finally, the probability $P_{s}=P_{s}^{1}+P_{s}^{2}+2 P_{s}^{3}+P_{s}^{4}$. The factor of 2 before $P_{s}^{3}$ accounts for the fact that the associated event results in the successful delivery of two packets to the AP.

The average length of a randomly chosen slot time is given by $T_{s}=\left(1-\tau_{s}\right)^{N}\left(1-\tau_{r}\right) \sigma+\left[\tau_{r}+\right.$ $\left.\pi_{r}(e)\left(1-\left(1-\tau_{s}\right)^{N}-N \tau_{s}\left(1-\tau_{s}\right)^{N-1} P_{s A}^{\text {out }}\left(1-P_{s R}^{\text {out }}\right)\right)\right] T_{b}+$ $2 \pi_{r}(t) T_{b}$, which accounts for the three possible slot durations. 1) Idle slots of duration $\sigma$, in which neither the relay nor any other node attempts to transmit a packet, thus having a probability $\left(1-\tau_{s}\right)^{N}\left(1-\tau_{r}\right)$. 2) Busy slots of duration $T_{b}$ in which either the relay transmits or a source node transmission is not followed by a relay transmission. The relay transmits if its backoff counter reaches zero, which occurs with probability $\tau_{r}$. A source node transmission is not followed by a relay transmission when the relay queue is empty (which occurs with probability $\left.\pi_{r}(e)\right)$ and no arrivals to the relay occur during source transmission. An arrival to the relay occurs when a source node transmission fails to reach the destination (due to channel outage) but reaches the relay, this event has a probability $N \tau_{s}\left(1-\tau_{s}\right)^{N-1} P_{s A}^{\text {out }}\left(1-P_{s R}^{\text {out }}\right)$. The event of no relay arrival is then the complement of that event, and we must take care to exclude the event of no source transmissions from that complement event. 3) Busy slots of duration $2 T_{b}$, in which a relay transmission follows a source node transmission, hence the factor of 2, such an occurs with probability $\pi_{r}(t)$.

Based on an 11 Mbps transmission rate, and payload of length $L=2312$ octets, typical slot duration are $\sigma=20 \mu \mathrm{s}$, $T_{p}=1681.5 \mu s$, and $T_{b}=2160.4 \mu s$.

\section{B. Delay}

In the proposed cooperation protocol, a packet can encounter two queuing delays; the first in the source node's queue and the second in the relay's queue. If a packet successfully transmitted by a source node arrives to the AP, then this packet is not stored on the relay's queue. Let $P_{a}$ denote the probability of this event. Then the total delay encountered by a packet can be modeled as

$$
D= \begin{cases}D_{s}, & \text { w.p. } P_{a} \\ D_{s}+D_{r}, & \text { w.p. } 1-P_{a}\end{cases}
$$

where $D_{s}$ and $D_{r}$ are the queuing delays in the source and relay queues, respectively. We can elaborate more on (16) as follows. For a given packet in the source node's queue, if the packet is delivered from the source to the AP directly (without relay help), then the delay encountered by this packet is only the queuing delay in the source nodes queue. On the other hand, if the packet is delivered to the AP through the relay, then the packet will encounter the following delays: queuing delay in the source nodes queue in addition to the queuing delay in the relays queue.

First, we find the queuing delay in either the source node or the relay queue, as both are modeled as M/G/1 queues, with the difference being in the average arrival and departure rates. For an M/G/1 queue, the mean waiting time in queue is given by the Pollaczek-Kinchin formula [16], $\mathrm{E}\left[W_{i}\right]=\frac{\lambda_{i} \mathrm{E}\left[S_{i}^{2}\right]}{2\left(1-\lambda_{i} / \mu_{i}\right)}$, where $i \in(s, r), \lambda_{i}$ is the average arrival rate, $\mu_{i}$ the average service rate, and $S_{i}$ the service time. From the mean waiting time, one immediately gets the mean queuing delay as $D_{i}=$ $\mathrm{E}\left[W_{i}\right]+\mathrm{E}\left[S_{i}\right]$.

The probability $P_{a}$, that, for any packet, the first successful transmission from the source node's queue is to the AP is given by $P_{a}=\frac{1-P_{s A}^{\text {out }}}{\left(1-P_{s A}^{\text {out }}\right)+\left(1-P_{s R}^{\text {out }}\right)-\left(1-P_{s A}^{\text {out }}\right)\left(1-P_{s R}^{\text {out }}\right)}$. And the average delay is thus given by $D=D_{s}+\left(1-P_{a}\right) D_{r}$.

\section{RESUlTS AND DISCUSSIONS}

We compare the performance of the cooperative protocol and the CSMA/CA protocol without cooperation. We set the SNR threshold $\beta=15 \mathrm{~dB}$ and the path loss exponent $\gamma=3.7$. The distance between any node and AP is 120 $\mathrm{m}$, and between any node and the relay $70 \mathrm{~m}$, and between relay and AP $50 \mathrm{~m}$. Transmission power is $100 \mathrm{~mW}$, and noise variance $N_{0}=10^{-11}$. Source node's initial contention window $W_{0}^{s}=32$ with $m=5$ backoff stages, and relay node's contention window size $W_{0}^{r}=32$. To validate the model, we have built a custom packet-based simulator, that closely follows all the IEEE 802.11 protocol details for each source node, and follows the details of our proposed protocol at the relay node. Simulation results are based on an $11 \mathrm{Mbps}$ transmission rate, and payload of length $\mathrm{L}=2312$ octets, slot duration $\sigma=20 \mu \mathrm{s}$, DIFS $=128 \mu \mathrm{s}$, and ACK_Timeout $=300 \mu \mathrm{s}$. 


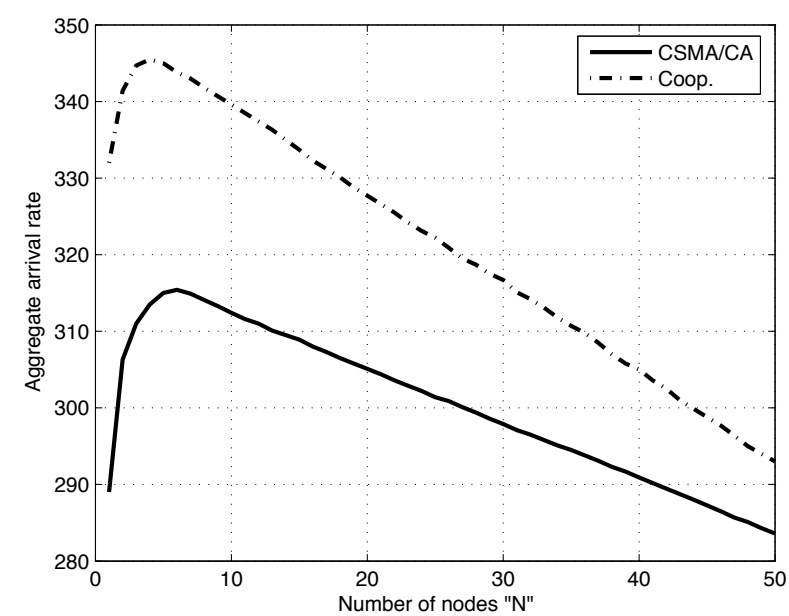

Fig. 4. Maximum achievable aggregate arrival rate vs number network nodes.

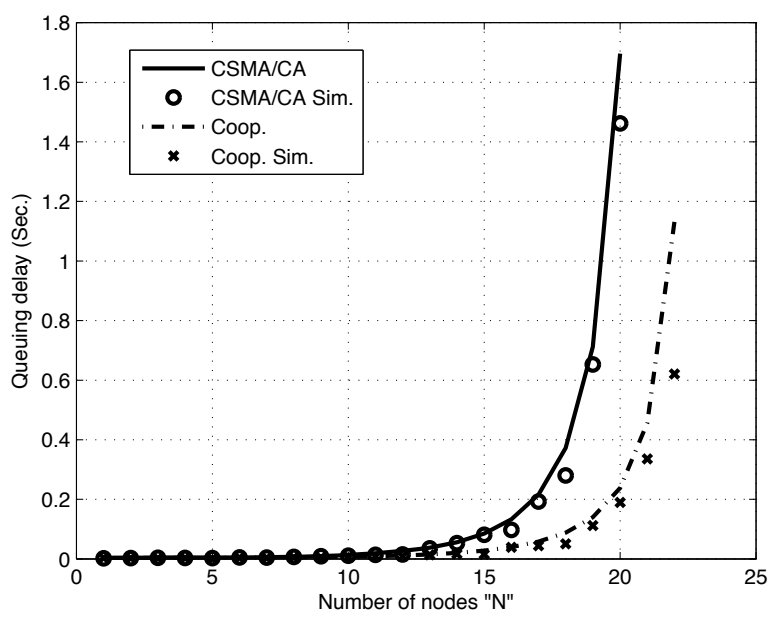

Fig. 5. Queuing delay vs. number of nodes for $\lambda_{s}=15$.

Fig. 4 depicts the maximum aggregate arrival rate (sum of the arrival rates of all network nodes) supported by the network while maintaining queues stability versus the number of network nodes. We can observe that, for a given number of nodes, the proposed cooperative protocols resulted in a $7 \%$ average increase in the maximum supported aggregate arrival rate. This increase is due to the fact that the relay node provides a more reliable path to the AP leading to a higher packet delivery rate. Therefore, source nodes are able to empty their queues at a faster rate, thus, freeing the channel for relay access, and for additional nodes that the network might accommodate. As the number of nodes increase the supported arrival rates start to decrease since the network starts to get congested and the queues' stability can no longer be supported without a decrease in arrival rates.

Fig. 5 shows the delay performance of our cooperative protocol compared to the non-cooperative CSMA/CA protocol. It is clear that our protocol outperforms CSMA/CA in terms of queuing delay. This is mainly because most of the relay's transmission attempts are made just after source nodes' transmissions, and not by waiting for the backoff counter to reach 0 . Therefore, the relay is guaranteed a high degree of

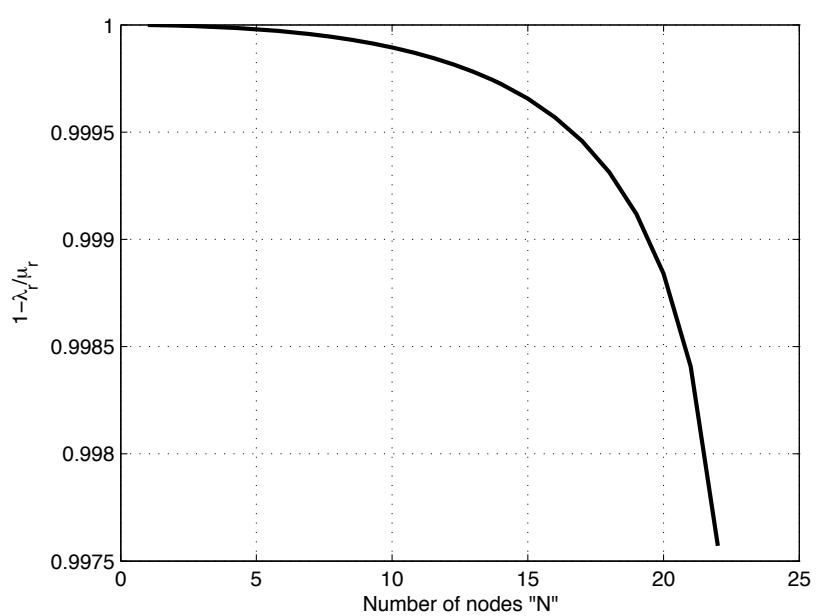

Fig. 6. Probability that relay queue is empty vs. number of nodes for $\lambda_{s}=$ 15.

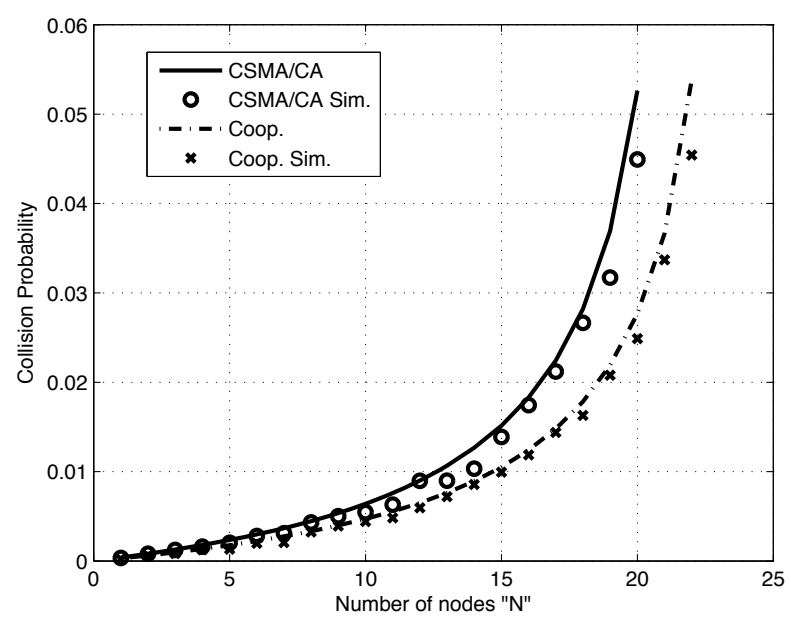

Fig. 7. Collision probability vs. number of nodes for $\lambda_{s}=15$.

uncontested channel access. Moreover, as the network load increases, the average number of source nodes' transmission attempts increase, which offers the relay more channel access opportunities to service its queue that now has a higher arrival rate. To prove this, the quantity $\left(1-\lambda_{r} / \mu_{r}\right)$, which from queuing theory is the probability that the relay queue is empty, is plotted in Fig. 6. It can be seen that there is less than $1 \%$ variation in the probability over the range of supported number of nodes.

Fig. 7 compares between the collision probability of CSMA/CA and our cooperative protocol. Another merit of our cooperation protocol and its channel access mechanism is that, the introduction of the relay node in the network does not result in an increased collision probability as it is the case with any random access protocol. We further notice a decrease in the collision probability, which is because of the second path to the AP the relay offers to the network nodes. This second path helps the different nodes empty their queues at a faster rate, hence, nodes do not have to access the channel as often as in the case without cooperation, which reduces the collision probability. Finally, Fig. 8 demonstrates the effect of cooper- 


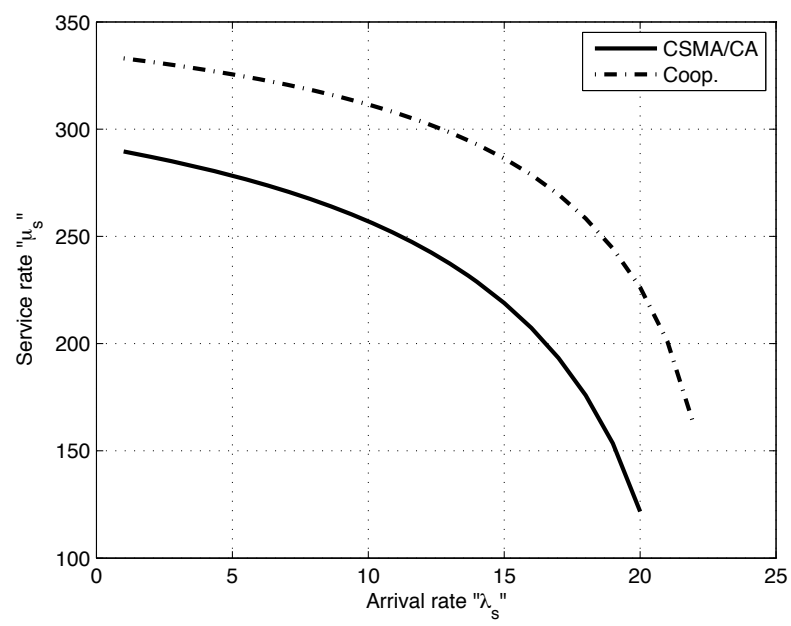

Fig. 8. Source node's service rate vs. arrival rate for $N=15$.

ation on how fast nodes' queues get empty by comparing the source node's service rates under both cooperative and noncooperative protocols. An average increase of $28 \%$ is observed in the service rate, which interprets the reduction achieved in the collision probability.

\section{CONCLUSIONS}

In this paper, we have proposed a novel cooperative protocol for IEEE 802.11 based wireless random access networks. Through cooperation, the proposed protocol mitigates the detrimental effects of wireless channel errors on the performance of CSMA/CA random access protocol. Cooperation is achieved by deploying a relay node that will help different network nodes to forward their packets to the AP. By virtue of the relay's channel access mechanism, the increase in collision probability associated with the addition of more nodes to the network is mitigated.

The protocol's performance is thoroughly investigated and compared to the non-cooperative CSMA/CA protocol. Performance characterization is achieved through the development of a Markov model coupled with queuing analysis of the network operation. The developed Markov model accurately described the network dynamics in the presence of relay, and captured the interactions between different network nodes. Results revealed a significant improvement in terms of the maximum achievable arrival nodes's rates, delay, and the number of nodes supported by the network.

\section{REFERENCES}

[1] J. N. Laneman, D. N. C. Tse, and G. W. Wornell, "Cooperative diversity in wireless networks: efficient protocols and outage behavior," IEEE Trans. Inf. Theory, vol. 50, pp. 3062-3080, Dec. 2004.

[2] W. Su, A. K. Sadek, and K.J.R. Liu, "Cooperative communications in wireless networks: Performance analysis and optimum power allocation," Wireless Personal Communications, vol. 44, no. 2, pp. 181-217, Jan. 2008.

[3] K. J. R. Liu, A. K. Sadek, W. Su, and A. Kwasinski, Cooperative Communications and Networking, Cambridge University Press, 2008.

[4] A. K. Sadek, K. J. R. Liu, and A. Epheremides, "Cognitive multiple access via cooperation: Protocol design and performance analysis," IEEE Trans. Inf. Theory, vol. 53, no. 10, pp. 3677-3696, Oct. 2007.
[5] A. A. El-Sherif, A. Kwasinski, A. K. Sadek, and K. J. R. Liu, "Content-aware multiple access protocol for cooperative packet speech communications," IEEE Trans. Wireless Commun., vol. 8, no. 2, pp. 995-1005, Feb. 2009.

[6] R. Lin and A. P. Petropulu, "A new wireless medium access protocol based on cooperation," IEEE Trans. Signal Process., vol. 52, no. 12, pp. 4675-4684, Dec. 2005.

[7] M. K. Tsatsanis, R. Zhang, , and S. Banerjee, "Network-assisted diversity for random access wireless networks," IEEE Trans. Signal Process., vol. 48, pp. 702-711, March 2000.

[8] IEEE Computer Society LAN MAN Standards Committee, Wireless LAN medium access control (MAC) and physical layer (PHY) specifications, IEEE Standard 802.11-1999, New York, NY: IEEE, 1999.

[9] P. Chatzimisios, A.C. Boucouvalas, and V. Vitsas, "Performance analysis of ieee $802.11 \mathrm{dcf}$ in presence of transmission errors," in Proc. Intl. Conf. on Comm. (ICC), June 2004, pp. 3854-3858.

[10] Y. Zheng, K. Lu, D. Wu, and Y. Fang, "Performance analysis of ieee 802.11 dcf in imperfect channels," IEEE Trans. Veh. Technol., vol. 55, no. 5, pp. 1648-1656, Sep. 2006.

[11] G. Bianchi, "Performance analysis of the ieee 802.11 distributed coordination function," IEEE J. Sel. Areas Comm., vol. 18, no. 3, pp. 535-547, Mar. 2000.

[12] Y. Xiao, "Saturation performance metrics of the 802.11 mac," in Proc. IEEE Veh. Technol. Conf., Oct. 2003, vol. 3, pp. 1453-1457.

[13] K. Duffy, D. Malone, and D. J. Leith, "Modelling of 802.11 distributed coordination function in non-saturated condition," IEEE Commun. Lett., vol. 9, no. 8, pp. 715-718, Aug. 2005.

[14] D. Malone, K. Duffy, and D. Leith, "Modelling the 802.11 distributed coordination function in nonsaturated heterogeneous conditions," IEEE/ACM Trans. Netw., vol. 15, no. 1, pp. 159-172, Feb. 2007.

[15] G. R. Grimmett and D. R. Stirzaker, Probability and Random Processes, Oxford University Press, 2001.

[16] Ronald W. Wolff, Stochastic Modeling and the Theory of Queues, Prentice Hall, 1989.

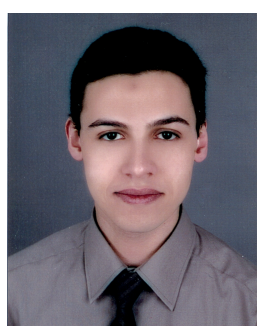

Amr El-Sherif (S' 00, M' 08) received the B.Sc. degree (with highest Honors) and the M.Sc. degree in electrical engineering form Alexandria University, Alexandria, Egypt in 2002 and 2005, respectively. $\mathrm{He}$ received the Ph.D. degree in electrical engineering from the University of Maryland, College Park, in 2009.

$\mathrm{He}$ is currently an Assistant Professor in the Electrical Engineering Department at Alexandria University, Egypt. His research interests include cooperative communications and networking, crosslayer design for wireless networks, multiple access technologies for wireless and sensor networks, and spectrum sharing and cognitive radio systems.

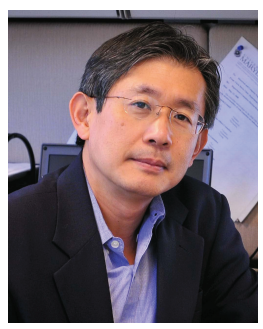

K. J. Ray Liu (F'03) is named a Distinguished Scholar-Teacher of University of Maryland, College Park, in 2007, where he is Christine Kim Eminent Professor in Information Technology. He serves as Associate Chair of Graduate Studies and Research of Electrical and Computer Engineering Department and leads the Maryland Signals and Information Group conducting research encompassing broad aspects of wireless communications and networking, information forensics and security, multimedia signal processing, and biomedical engineering.

Dr. Liu is the recipient of numerous honors and awards including IEEE Signal Processing Society Technical Achievement Award and Distinguished Lecturer. He also received various teaching and research recognitions from University of Maryland including university-level Invention of the Year Award; and Poole and Kent Senior Faculty Teaching Award and Outstanding Faculty Research Award, both from A. James Clark School of Engineering. An ISI Highly Cited Author in Computer Science, Dr. Liu is a Fellow of IEEE and AAAS.

Dr. Liu is President and was Vice President - Publications of IEEE Signal Processing Society. He was the Editor-in-Chief of IEEE Signal Processing Magazine and the founding Editor-in-Chief of EURASIP Journal on Advances in Signal Processing. 